\title{
A LAW OF THE ITERATED LOGARITHM FOR STABLE SUMMANDS
}

\author{
JOSHUA CHOVER ${ }^{1}$
}

Let $x_{n}(n=1,2,3, \cdots)$ be mutually independent random variables, identically distributed according to the symmetric stable distribution with exponent $\gamma(0<\gamma \leqq 2)$, i.e., $E\left[\exp \left(i t x_{n}\right)\right]=\exp (-|t| \gamma)$. Let $S_{n}=\sum_{k=1}^{n} x_{k}$. The classical "law of the iterated logarithm" (for the simplest exposition, see Feller [2, pp. 192-195]; see also [3] and [4]) tells us that for $\gamma=2$

$$
P\left(\limsup _{n \rightarrow \infty} \frac{S_{n}}{\sqrt{ }(2 n \log \log n)}=1\right)=1 .
$$

That is, the variables $(1 / \sqrt{ } n) S_{n}$ again satisfy $E\left[\exp \left(i t(1 / \sqrt{ } n) S_{n}\right)\right]$ $=\exp \left(-|t|^{2}\right)$, and to achieve a finite lim sup they must be cut down additionally (and multiplicatively) by the factors $(2 \log \log n)^{-1 / 2}$. For some reason the obvious corresponding statement for the case $\gamma<2$ does not seem to have been recorded, and it is the purpose of this note to do so.

For $0<\gamma<2$, the variables $n^{-\gamma^{-1}} S_{n}$ again satisfy $E\left[\exp \left(i t n^{-\gamma^{-1}} S_{n}\right)\right]$ $=\exp (-|t| \gamma)$. Since the corresponding distribution function $F(x)$ has tail behavior $F(-x)+1-F(x) \sim$ (const) $|x|^{-\gamma}$ as $|x| \rightarrow \infty$, instead of exponential decrease as in the $\gamma=2$ case, we can expect the "cut down factors" to appear otherwise than as multipliers.

TheOREM. For $\gamma<2$

$$
P\left(\limsup _{n \rightarrow \infty}\left|n^{-\gamma^{-1}} S_{n}\right|^{(\log \log n)^{-1}}=e^{\gamma^{-1}}\right)=1 .
$$

We sketch the proof. It suffices to show that for fixed $\epsilon>0$, and for almost every sample point, we have

$$
\left|n^{-\gamma^{-1}} S_{n}\right|>(\log n)^{(1+\epsilon) \gamma^{-1}} \quad \text { finitely often }
$$

and

$$
\left|n^{-\gamma^{-1}} S_{n}\right|>(\log n)^{(1-e) \gamma^{-1}} \quad \text { infinitely often. }
$$

Now the proof proceeds almost exactly as for the $\gamma=2$ case. Thus, to show (1), let $A_{n}$ be the event that $\left|S_{n}\right|>n^{\gamma^{-1}}(\log n)^{(1+\epsilon) \gamma^{-1}}$. Pick

Received by the editors June 1, 1965.

1 Research supported by NSF Grant GP-3483. 
$\beta>1$, and for $r=1,2,3, \cdots$, let $n_{r}$ denote $\left[\beta^{r}\right]$, the largest integer in $\beta^{r}$. Let $B_{r}$ denote the event that $\left|S_{n}\right|>\left(n_{r}\right)^{\gamma^{-1}}\left(\log n_{r}\right)^{(1+\epsilon) \gamma^{-1}}$ for some $n$ with $n_{r} \leqq n<n_{r+1}$. Then $\lim \sup _{n \rightarrow \infty} A_{n} \subset \lim \sup _{r \rightarrow \infty} B_{r}$; and there exists a constant $c>0$ independent of $r$ such that for all $r P\left(B_{r}\right)$ $\leqq c P\left(C_{r}\right)$, where $C_{r}$ is the event that

$$
\left(n_{r+1}-1\right)^{-\gamma^{-1}}\left|S_{n_{r+1}-1}\right|>\left(\frac{n_{r}}{n_{r+1}-1}\right)^{\gamma^{-1}}\left(\log n_{r}\right)^{(1+e) \gamma^{-1}} .
$$

Since the distribution for $\left(n_{r+1}-1\right)^{-\gamma^{-1}} S_{n_{r+1}-1}$ has tail behavior $\sim$ (const) $|x|^{-\gamma}$ (cf. [3, pp. 181-182]), we conclude that for some finite constant $a>0, P\left(C_{r}\right) \leqq a r^{-(1+\epsilon)}$, and $\sum_{r} P\left(B_{r}\right)<\infty$. Hence by the Borel Cantelli lemma, $P\left(\lim \sup _{n \rightarrow \infty} A_{n}\right)=P\left(\lim \sup _{r \rightarrow \infty} B_{r}\right)=0$, and (1) holds.

To prove (2), set $D_{r}=S_{n_{r+1}}-S_{n_{r}}$. These are independent variables, and by the Borel Cantelli lemma again we find that for almost every sample point,

$$
\left(n_{r+1}-n_{r}\right)^{-\gamma^{-1}}\left|D_{r}\right| \geqq\left(\log n_{r}\right)^{\left(1-\frac{1}{\epsilon} \epsilon\right) \gamma^{-1}}
$$

for infinitely many $r$. Suppose that (2) does not hold on a set of positive probability. Then for almost every sample point in that set,

$$
\begin{aligned}
&\left|n_{r+1}^{-\gamma^{-1}} S_{n_{r+1}}\right| \geqq\left(1-\frac{n_{r}}{n_{r+1}}\right)^{\gamma^{-1}}\left|D_{r}\right|-\left(\frac{n_{r}}{n_{r+1}}\right)^{\gamma^{-1}}\left|S_{n_{r}}\right| \\
& \geqq\left(1-\frac{n_{r}}{n_{r+1}}\right)^{\gamma^{-1}}\left(\log n_{r}\right)^{\left(1-\frac{1}{\epsilon} \epsilon\right) \gamma^{-1}}-\left(\frac{n_{r}}{n_{r+1}}\right)^{\gamma^{-1}}\left(\log n_{r}\right)^{(1-\epsilon) \gamma-1}
\end{aligned}
$$

for infinitely many $r$. But for large $r$ the last difference in (3) dominates $\left(\log n_{r+1}\right)^{(1-\epsilon) \gamma^{-1}}$; so (2) does hold almost everywhere. For further details in this paraphrase of the classical case, we refer the reader to Feller [2], loc. cit.

REMARK. By stricting $n$ to subsequences of the form $n_{k}=\left[\beta^{k^{8}}\right]$ for fixed $\beta>1$ and $\delta>1$, the proof shows that, with probability 1 , every point in the interval $\left[1, e^{\gamma^{-1}}\right]$ is a limit point of the sequence $\left\{n^{-\gamma^{-1}}\left|S_{n}\right|^{(\log \log n)^{-1}}, n=1,2,3, \cdots\right\}$. Now, at least for $1<\gamma<2$, 0 is also a limit point, as one can conclude from the general results of Chung and Fuchs (see [1, Theorem 4]). I do not know about the points in the interval $(0,1)$.

Added in proof. V. Strassen has pointed out to us that the above theorem follows simply from a result of $\mathrm{A}$. Khinchine, Mat. Sb. 45 (1938) ; p. 582. However, the present version of the log log law holds also if the common d. f. $F$ of the $x_{n}$ lies in that part of the domain of 
normal attraction of a nonnormal stable d.f. $G_{\gamma}(0<\gamma<2)$ subject to conditions of the form

$$
F(-x)=\frac{c_{1}}{x^{\gamma}}+\frac{d_{1}}{x^{\delta}}+r_{1}(x), \quad 1-F(x)=\frac{c_{2}}{x^{\gamma}}+\frac{d_{2}}{x^{\delta}}+r_{2}(x),
$$

where $r_{i}(x)=O\left(1 / x^{\epsilon}\right)$ and $\gamma<\delta<\epsilon$ (and $r_{1}(x)+r_{2}(x)$ are monotone as $x \rightarrow \infty$ if $\gamma<1)$. For under these conditions, H. Cramer has shown (On asymptotic expansions for sums of independent random variables with a limiting stable distribution, Sankhya Ser. A 25 (1963), 12-24) that for the d.f. $F_{n}$ of $S_{n}$ (suitably shifted and scaled), $F_{n}(x)-G_{\gamma}(x)$ $=O\left(1 / n^{\gamma}\right)$ uniformly in $x$. Hence in the above proofs, we may replace tail estimates based on $F_{n}$ by ones based on $G_{\gamma}$ with an error of at most $O\left(1 / n^{\gamma}\right)$. But on subsequences $n_{j} \subset\left[c^{j}\right], c>1$, such errors will not affect the convergence or divergence of our series, and the proofs go through as before.

\section{REFERENCES}

1. K. L. Chung and W. H. J. Fuchs, On the distribution of sums of random variables, Mem. Amer. Math. Soc., No. 6 (1951).

2. W. Feller, An introduction to probability theory and its applications. I, 2nd ed., Wiley, New York, 1957.

3. - The general form of the so-called law of the iterated logarithm, Trans. Amer. Math. Soc. 54 (1963), 373-402.

4. - A limit theorem for random variables with infinite moments, Amer. J. Math. 68 (1946), 257-262.

5. B. V. Gnedenko and A. N. Kolmogorov, Limit distributions for sums of independent random variables (Transl. by K. L. Chung), Addison-Wesley, Reading, Mass., 1954.

UNIVERSITY OF WISCONSIN 